\title{
Intermittent and Mild Persistent Asthma in Young Children, Adolescents and Adults: What Treatment Options?
}

\section{Cheepsattayakorn $\mathbf{A}^{1,2, *}$ and Cheepsattayakorn $\mathbf{R}^{3}$}

$110^{\text {th }}$ Zonal Tuberculosis and Chest Disease Center, Chiang Mai, Thailand

${ }^{2} 5^{\text {th }}$ Office of Disease Prevention and Control, Department of Disease Control,

Ministry of Public Health, Thailand

${ }^{3}$ Department of Pathology, Faculty of Medicine, Chiang Mai University, Chiang Mai, Thailand

*Corresponding author: Attapon Cheepsattayakorn, 10 $0^{\text {th }}$ Zonal Tuberculosis and Chest Disease Center, 143 Sridornchai

Road Changklan Muang Chiang Mai 50100 Thailand, Tel : 6653 140767, 6653 276364, Fax : 6653 140773, 66 53

273590, Email : Attapon1958@gmail.com, attaponche@yahoo.com

\section{Editorial}

Matching the treatment intensity with the severity of asthmatic symptoms is the basic treatment for asthma. Intermittent treatment with rapid symptom relief should be administered in asthmatic patients with infrequent and mild symptoms whereas a long-term controller medication should be given to patients with mild, persistent symptoms. Oral glucocorticoids requirement are encouraged to take daily controller medication to prevent asthmatic attacks in patients with mild, intermittent symptoms whose asthma often occur more than two times per year. Ongoing patient education, effective communication, and regular reassessment of asthma control are critical for long-term success in all types of asthma. A recent study demonstrated that asneeded use of acetaminophen was not associated with a higher incidence of asthma exacerbations or worse asthma control than was as-needed use of ibuprofen among young children with mild, persistent asthma. 